\title{
Temperature Effects in the Photoluminescence of Semiconductor Quantum Dots
}

\author{
Anatoly Zatsepin and Dmitry Biryukov
}

\begin{abstract}
Temperature effects in the exciton photoluminescence specific to semiconductor quantum dots (QDs) are reviewed using Si QDs as an example. The processes of direct and indirect optical excitation of spatially confined excitons in quantum dots embedded in dielectric matrix are analyzed. The temperature behavior of the quantum dots photoluminescence (PL) excited by various methods was described in detail by a generalized electronic transitions scheme using different exciton relaxation models. The different types of temperature dependences were analyzed. The analytical expressions were obtained for their description, which allow one to determine the energy and kinetic characteristics of QD photoluminescence. It was found that the shape of the temperature dependence makes it possible to understand whether the process of exciton relaxation contains several different thermally activated stages or this is a simple one-stage process. The applicability of the obtained expressions for the analysis of the luminescence properties of quantum dots is demonstrated by the example of crystalline and amorphous silicon nanoclusters in silica matrix. It has been established that the quantum confinement effect of excitons in quantum dots leads to a decrease in the frequency characteristics and thermal activation barriers for nonradiative transitions.
\end{abstract}

Keywords: quantum dots, exciton photoluminescence, temperature dependence of luminescence, quantum confinement effects, ion implantation, mechanisms of excitation and relaxation

\section{Introduction}

The growing interest in the optical properties of low-dimensional systems is stimulating the development of next-generation solid-state devices in the fields of photonics, microelectronics, and optoelectronics. In particular, various technological developments use semiconductor quantum dots formed inside a dielectric matrix [1-3]. It is well known that the luminescent activity and other physical properties of such materials are largely dependent on the transformation of the electron energy spectrum of QDs caused by the size factor [1, 2]. Moreover, the photoluminescence efficiency of QDs is also affected by the mechanisms of optical excitation and the effects of quantum confinement of excitons.

According to the results of many studies [4-14], the temperature dependences of photoluminescence in dielectric and semiconductor nanostructures can 
significantly differ in shape and type when using various excitation methods. The PL temperature curves of quantum dots are most often presented in the form of decreasing functions with increasing temperature $[4,5,7]$. At the same time, curves with a clearly defined maximum or a monotone increase in PL intensity is sometimes observed. This is most often characteristic of direct luminescence excitation of confined excitons [9-13]. The energy transfer of emitting nanoparticles through intermediate electronic states of the matrix [4-7] can also lead to an increase in PL intensity and to curves with an extreme shape. Thus, there is a need for a detailed analysis of various energy transfer schemes and types of electronic transitions in order to explain the observed variety of forms of temperature dependences of QD photoluminescence [4-13].

It should also be said that according to some researchers, the spectral parameters of most luminescent low-scale structures are largely determined by sized and geometric factors $[12,15,16]$. In this case, the shape of the temperature curves of quantum dots PL is also substantially transformed [12]. This suggests that information on the features of the confined excitons in quantum dots can be obtained by analyzing the temperature behavior of photoluminescence. However, the lack of systematic research in this field leaves this question open.

The purpose of this chapter is to analyze the luminescence temperature dependences under direct and indirect optical excitation of spatially confined excitons in QDs. A generalized analytical description of such functional dependences is also reported.

\section{Thermal and ion beam formation quantum dots}

Obtaining methods of semiconductor quantum dots are questions of great importance in the fields of science and engineering. Numerous advances have been achieved in the synthesis of QDs, among them bulk etching [17], laser pyrolysis [18], gas phase synthesis [19], thermal vaporization [20], and wet chemistry techniques [21]. It should be noted that all of the above methods often require special additives such as surfactants and dopants, as well as high-temperature postprocessing, to stabilize the QDs and control their size.

Another convenient method is the thermal synthesis of quantum dots. As shown in [22-24], the synthesis of silicon QDs in the suboxide matrix takes place according to a reaction, which, depending on the temperature and duration of annealing, can be stopped at any intermediate stage: $2 \mathrm{SiO}_{\mathrm{X}} \rightarrow \mathrm{Si} \mathrm{QD}+\mathrm{SiO}_{2}$.

The schematic representation of the sample transformation is shown in Figure 1.

With ion implantation, direct accumulation of the introduced material with not only the quantum dots formation but also the quantum dots occurrence is possible due to the evolution of defective structures. In particular, there is an innovative method of creating $\mathrm{Si}$ quantum dots in $\mathrm{SiO}_{2}$ under pulsed ion beam exposure [28]. In this case, quantum dots are formed as a result of the conversion and clustering of radiation defects: ODC(II) $\rightarrow \mathrm{E}^{\prime} \rightarrow$ ODC(I) $\rightarrow$ Si QDs. During Gd-ion implantation with different doses, $\mathrm{Si}-\mathrm{O}$ bond softening appeared, and the three main stages of defect evolution were identified: (A) formation of primary oxygen-deficient centers; (B) conversion of defects; and (C) clustering into Si QDs (Figures 2 and 3).

By changing the modes of radiation exposure of the $\mathrm{SiO}_{2}$ matrix, we can control the qualitative and quantitative composition of defects and modify the optical properties of the host, including the ultraviolet transmittance and visible luminescence. As seen from Figure 2, the radiation defect conversion includes several consequent stages. The implementation of the described conversion mechanism provides the controlled formation of stable quantum dots at various modes of ion 


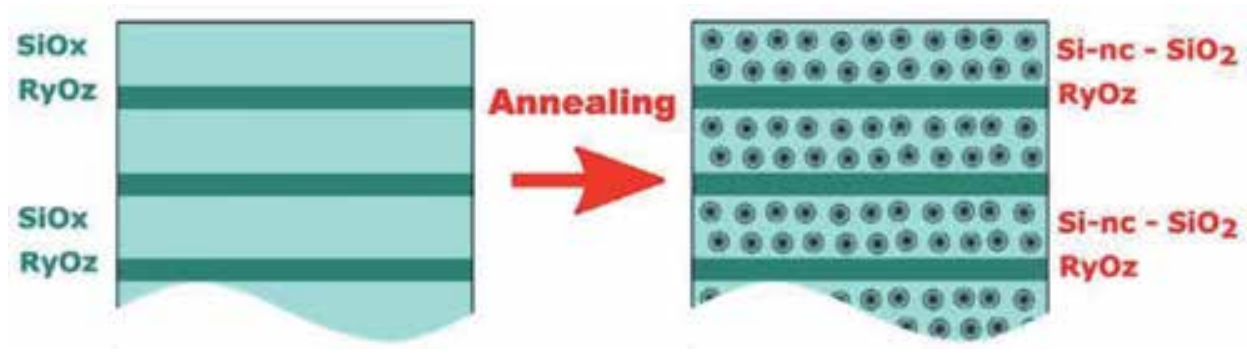

Figure 1.

Scheme of silicon nanoclusters (Si $Q D)$ formation in an initial multilayered structure under high-temperature annealing in nitrogen atmosphere. The $\mathrm{RyOz}(\mathrm{R}-\mathrm{Si}, \mathrm{Al}, \mathrm{Zr})$ is a dialectical layer.

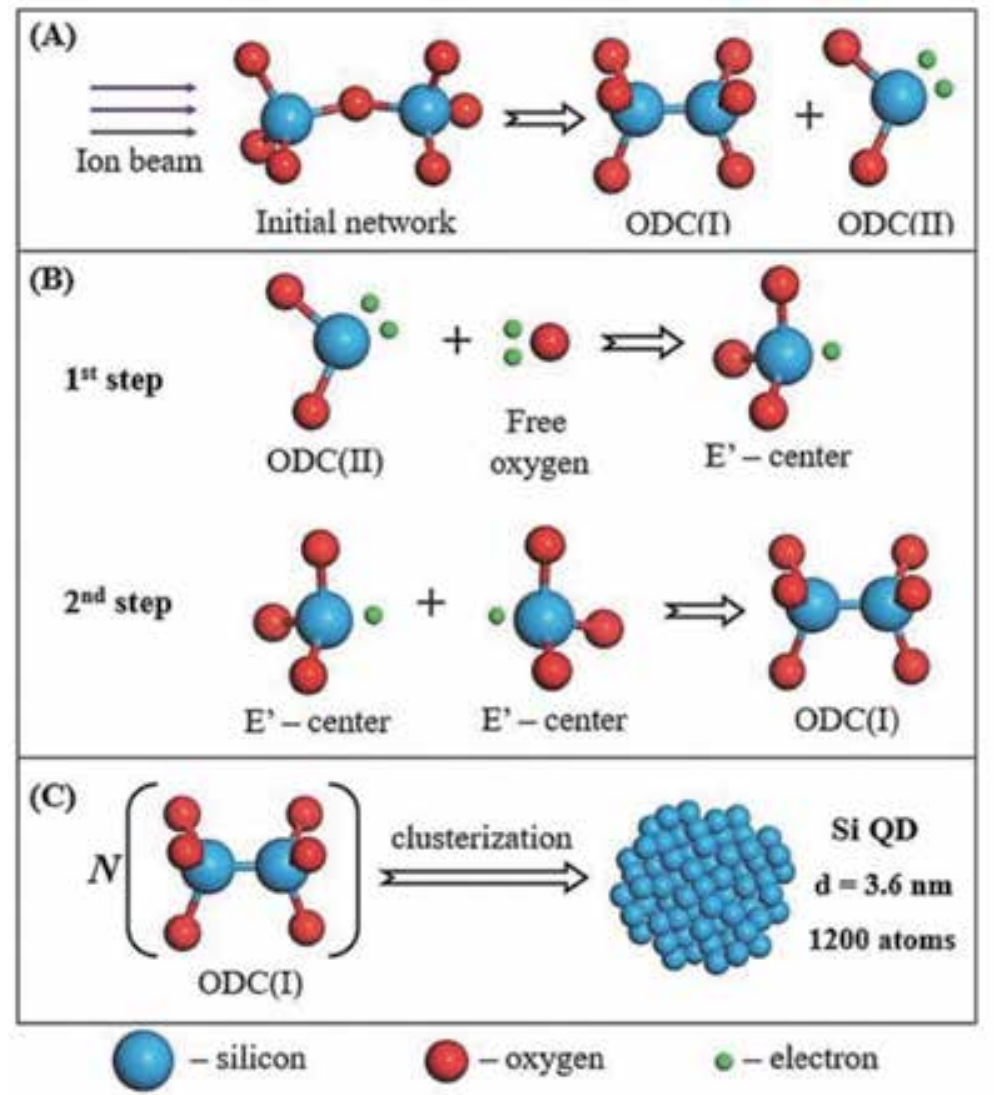

Figure 2.

Scheme of the successive stages for oxygen-deficient defect formation and fabrication of silicon quantum dots in $\mathrm{SiO}_{2}$ subjected to ion beam irradiation. (A) Radiation formation of vacancy defects; (B) transformation of defects; (C) clusterization into quantum dots.

implantation [25]. The resulting Si QDs have a relatively small size of about $3.6 \mathrm{~nm}$, which makes it possible to excite red luminescence due to the strong quantum confinement effect (Figure 3).

Described above the thermal synthesis of quantum dots and ion implantation methods are most convenient for stabilizing QDs in a dielectric matrix. Such systems are better suited for studying the mechanisms of excitation and radiative relaxation of QDs [26-28]. 


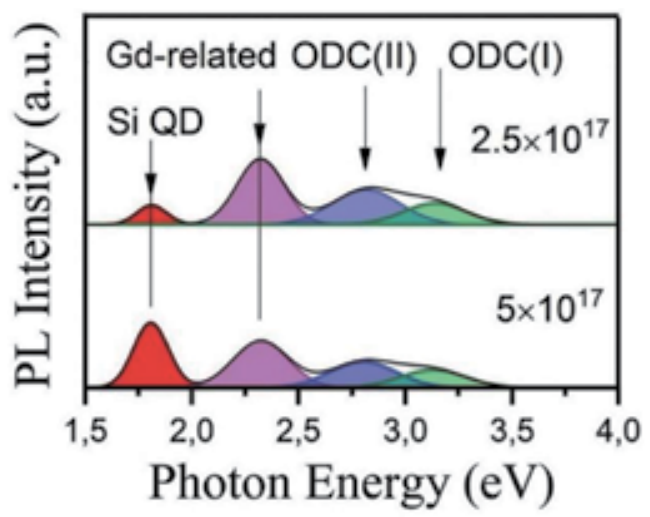

Figure 3.

Typical luminescence spectra of quantum dots and radiation defects in $\mathrm{SiO}_{2}$ implanted with $30 \mathrm{keV}$ Gd ions at fluences $\left(2.5 \times 10^{17}\right.$ and $\left.5 \times 10^{17} \mathrm{~cm}^{-2}\right)$. The spectra were obtained at temperature of $8 \mathrm{~K}$ and excitation by photons $6.6 \mathrm{eV}$.

\section{Mechanisms of QD excitation and scheme of electronic transitions}

In considering the intracenter relaxation processes for spatially confined excitons in QDs, their spin state should be taken into account. Bound electron-hole pair is a diamagnetic excitation characterized by singlet and triplet levels of energy associated with mutually antiparallel and parallel spins, respectively. In accordance with the well-known Hund's rule, the triplet state is the smallest excited state because the atomic level with lower energy has a full orbital angular momentum or maximum multiplexing $[29,30]$.

The lifetime of triplet excitations can be several orders of magnitude longer than that of the singlet one, because triplet-singlet radiative transitions are spin-forbidden [30]. The thermally activated character of triplet luminescence after direct excitation of the singlet state is due to the energy barrier between these terms. Therefore, a growth in temperature leads to an increase in the glow intensity [10-13].

A generalized diagram for electronic transitions is shown in Figure 4. Direct singlet-singlet PL excitation of the QDs is shown by transition 1. At the same time, transition 6 illustrates indirect PL excitation through the electronic states of the matrix.

Each of the three different models presented in this diagram is individually characterized by a small number of fitting parameters. For better understanding of these analytic calculations, we have made the following special notations:

$\Delta E_{I S C}=\left(E_{2}-E_{3}\right)$ denotes the energy factor of the singlet-triplet intersystem crossing (ISC) relative to the QD excitons.

$E_{Q}=E_{5}$ is the activation barrier of the QD PL quenching.

$E_{S T}=E_{8}$ is the activation barrier of self-trapping of matrix excitons.

$\Delta E_{O C}=\left(E_{11}-E_{12}\right)$ is the occupation energy factor of the radiative $\mathrm{T}_{1}$ triplet states of the QD exciton.

$\delta P_{I S C}=p_{03} / p_{02}$ is the intersystem crossing kinetic factor for spatially confined excitons in $\mathrm{QD}$.

$\delta P_{T}=p_{08} / P_{7(10)}$ is the energy transfer kinetic factor from the excitons of the matrix to the QDs.

$\delta P_{O C}=p_{012} / p_{011}$ is the occupation kinetic factor of the radiative $\mathrm{T}_{1}$ triplet states of the QD exciton.

$\delta P_{R}=p_{05} / P_{4}$ is the kinetic factor for the triplet-singlet radiative transition of the QDs. 


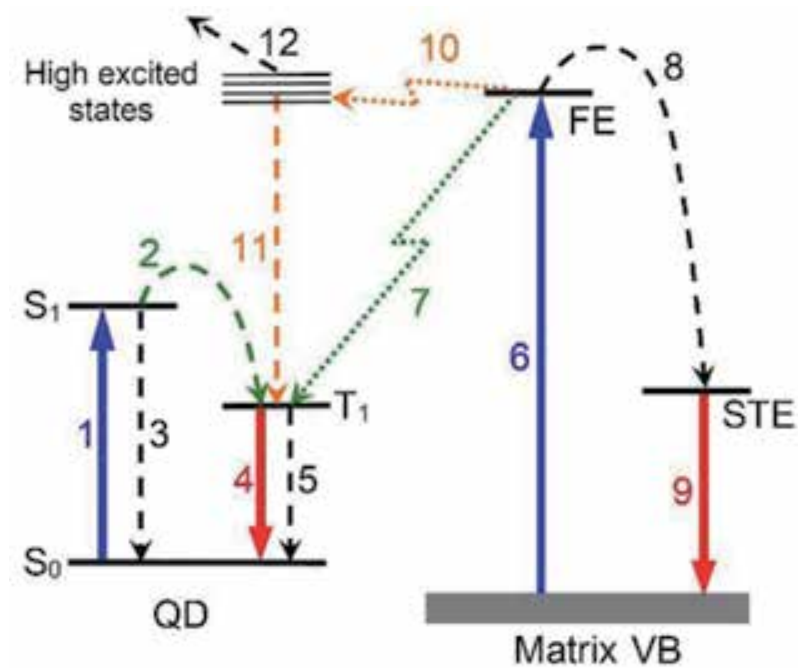

Figure 4.

Scheme of electron transitions in $Q D$ under direct and indirect excitations. Scheme shows two independent channels of excitation of the quantum dot and three different models of relaxation of excitons described by Eqs. (1)-(3). Optical and nonradiative transitions are indicated by solid and dashed arrows, respectively; the ground and excited singlet states are marked with $S_{o}$ and $S_{1}$, respectively; the excited triplet state of the exciton is $T_{1}$; the levels of free and self-trapped host matrix excitons are shown as FE and STE, respectively.

Here $E_{2}, E_{3}, E_{5}, E_{8}, E_{11}$, and $E_{12}$ are the thermal activation barriers. The frequency factors characterizing the nonradiative transitions $2,3,5,8,11$, and 12 are designated as $p_{02}, p_{03}, p_{05}, p_{08}, p_{011}$, and $p_{012}$, respectively; the singlet-triplet radiative transition rate and energy transfer rates corresponding to transitions 7 and 10 are denoted by $P_{4}, P_{7}$, and $P_{10}$, respectively.

In the work of [10], an analytical expression is presented that well describes the extreme form of the temperature dependence of PL. It was obtained taking into account the three-level energy scheme, which includes transitions 1-5. Process with an intersystem crossing (transition 2) and a triplet-singlet luminescence (transition 4) are the main stages of the two-stage radiative recombination of the excitons. Herewith the PL intensity will be proportional to the product of the quantum efficiencies $\eta_{I S C}$ and $\eta_{R}$ for transitions 2 and 4, respectively.

Given the above and skipping the intermediate stages, the final expression for the model of QDs direct excitation can be written in the following function form:

$$
I_{T}=I_{0} \eta_{\mathrm{ISC}} \eta_{\mathrm{R}}=I_{0}\left\{\left[1+\delta P_{\mathrm{ISC}} \exp \left(\frac{\Delta E_{\mathrm{ISC}}}{k T}\right)\right]\left[1+\delta P_{\mathrm{R}} \exp \left(-\frac{E_{\mathrm{Q}}}{k T}\right)\right]\right\}^{-1},
$$

where $I_{O}$ is the maximum luminescence intensity achieved at $\eta_{I S C}=1$ and $\eta_{R}=1$.

Eq. (1) has the four fitting parameters: $\delta P_{\text {ISC }}$ and $\Delta E_{\mathrm{ISC}}$ (transition 2) and $\delta P_{\mathrm{R}}$ and $E_{\mathrm{Q}}$ (transition 4). In expression (1) the physical meaning of the constants $\left(\Delta E_{\mathrm{ISC}}, \delta P_{\mathrm{ISC}}, E_{\mathrm{Q}}\right.$, and $\left.\delta P_{\mathrm{Q}}\right)$ is quite clear. The $\Delta E_{\mathrm{ISC}}$ and $\delta P_{\mathrm{ISC}}$ parameters characterize the occupation process for $\mathrm{T}_{1}$ triplet state, whereas $E_{\mathrm{Q}}$ and $\delta P_{\mathrm{Q}}$ are the parameters of the quenching process for the triplet-singlet PL. As can be seen from Eq. (1), an increase in the intensity of the triplet luminescence in the general process can be caused by the conversion of excitation from the singlet to the triplet state under some definite conditions. However, the shape of the temperature curve of the luminescence can significantly depend on various ratios between the activation energies $E_{2}$ and $E_{3}$. 
Other triplet PL relaxation schemes can be considered in the same way. The energy transfer of free matrix excitons (FE) to quantum dots is confirmed experimentally $[4,5,13]$. In other words, the absorption of high-energy light quanta with the formation of free excitons (Figure 4, transition 6) can excite luminescence of QDs. The direct transition of the excitation to the triplet state T1 (transition 7) illustrates the simplest scheme of this process. The indicated option is similar to the direct excitation of QDs described by a two-stage process [10,13]. The expression for the intensity of triplet luminescence (Eq. (2)) can be written assuming that transition 7 occurs without a barrier, and the formation of a self-trapped exciton (transition 8) is a competing process and also leads to luminescence (transition 9).

$$
I_{T}{ }^{\prime}=I_{0} \eta_{\mathrm{T}} \eta_{\mathrm{R}}=I_{0}\left\{\left[1+\delta P_{\mathrm{T}} \exp \left(-\frac{E_{\mathrm{ST}}}{k T}\right)\right]\left[1+\delta P_{\mathrm{R}} \exp \left(-\frac{E_{\mathrm{Q}}}{k T}\right)\right]\right\}^{-1},
$$

where $\eta_{R}$ and $\eta_{T}$ are quantum efficiencies characterizing radiative triplet-singlet transitions and energy transfer from FE to QDs, respectively. Eq. (2) has four fitting parameters: $\delta P_{\mathrm{R}}$ and $E_{\mathrm{Q}}$ (transition 4$)$ and $\delta P_{\mathrm{T}}$ and $\Delta E_{\mathrm{ST}}$ (transition 7), similar to Eq. (1). At the same time, the parameters $\left(\delta P_{\mathrm{R}}\right.$ and $\left.E_{\mathrm{Q}}\right)$ of nonradiative decay of excitons are independent of the excitation method, as can be seen from Eqs. (1) and (2). An analysis of the data for direct excitation of PL (Figure 4, transitions 4 and 5) allows one to determine these two parameters from independent measurements and then use them in the analysis of indirect excitation processes. Using this approach, one can significantly improve the procedure for approximating the experimental temperature dependences of PL and reduce the number of variable parameters.

At the same time, the large difference between the energy levels of excitons in QDs and free excitons in a dielectric matrix should be taken into account. In this regard, Eq. (2) is approximate and describes only a simplified scheme of luminescence. In fact, transition 7 cannot be considered elementary, and energy from the FE is transferred to the high-energy states (HES) of the QDs (see Figure 4, transition 10). Further, successive thermal transitions from HES lead to the filling of the lower triplet state T1. The indicated energy transfer sequence cannot be considered barrierfree, and triplet-singlet luminescence should be described by a multistage model.

Let us consider the generalized transition 11 with effective parameters: activation energy $E_{11}$ and frequency factor $p_{011}$ as a sequence of thermal transitions $\mathrm{HES} \rightarrow \ldots \rightarrow \mathrm{T}_{1}$. A generalized transition 12 with the corresponding effective parameters $E_{12}$ and $p_{012}$ will be considered a sequence of competing processes. The energy transfer rate $P_{10}$ to highly excited states will be assumed to be independent of temperature, and the thermal activation barrier of such a transfer is equal to zero. Then the triplet-singlet PL will be described by the following three-stage model:

$$
\begin{aligned}
I_{T^{\prime \prime}} & =I_{0} \eta_{\mathrm{T}} \eta_{\mathrm{oc}} \eta_{\mathrm{R}} \\
& =I_{0}\left\{\left[1+\delta P_{\mathrm{T}} \exp \left(-\frac{E_{\mathrm{ST}}}{k T}\right)\right]\left[1+\delta P_{\mathrm{oc}} \exp \left(\frac{\Delta E_{\mathrm{oc}}}{k T}\right)\right]\left[1+\delta P_{\mathrm{R}} \exp \left(-\frac{E_{\mathrm{Q}}}{k T}\right)\right]\right\}^{-1}
\end{aligned}
$$

The latter is taking into account the quantum efficiencies $\left(\eta_{T}, \eta_{o c}, \eta_{R}\right)$ for energy transfer, triplet state occupation, and radiative triplet-singlet transitions, respectively.

Eq. (3) is the variant of Eq. (2), where by taking into account an additional step there are six parameters, corresponding to transition 10 ( $\left.\delta P_{\text {Т и }} \Delta E_{\mathrm{ST}}\right)$, transition 11 $\left(\delta P_{\text {oc }}\right.$ и $\left.\Delta E_{\text {oc }}\right)$, and transition $4\left(\delta P_{\mathrm{R}}\right.$ и $\left.E_{\mathrm{Q}}\right)$. The energy transfer from the matrix to the quantum dot is denoted identically in Eqs. (2) and (3) and is described by the parameters $\delta P_{\mathrm{T}}$ and $\Delta E_{\mathrm{ST}}$. However, it should be clarified that in Eq. (2) quantum energy transfer efficiency $\eta_{\mathrm{T}}$ characterizes transition 7, while in Eq. (3)-transition 10. 
As can be seen from Figure 1, radiation transition 4 with fitting parameters $\left(\delta P_{\mathrm{R}}\right.$ и $\left.E_{\mathrm{Q}}\right)$ is a common step for all considered models. Thus, the constant values of the parameters $\delta P_{\mathrm{R}}$ и $E_{\mathrm{Q}}$ for transitions 4 under direct or indirect excitation will significantly reduce the arbitrariness of approximation by Eqs. (1)-(3).

\section{Dependence of PL temperature behavior on energy and kinetic factors}

As was mentioned in previous sections, the dependence of the PL intensity on temperature $I_{\mathrm{T}}(T)$ can be described by a decreasing or increasing function or a curve with an extremum. According to Eq. (1), the form of $I_{\mathrm{T}}(T)$ function depends on the relations between the kinetic and activation parameters of luminescent centers. Thus, if the certain conditions determining the form of function will be known, one can associate the shape of $I_{\mathrm{T}}(T)$ curve with the energy and vibrational characteristics of the emission center. In the framework of two-step PL mechanism, the shape of the curves $I_{T}(T)$ is determined by the multipliers $\eta_{I S C}(T)$ and $\eta_{R}(T)$. Figure 5 shows the calculated functions $\eta_{I S C}(T)$ and $\eta_{\mathrm{R}}(T)$ and their multiplication. The curve (3) reproduces qualitatively the temperature dependence $I_{T}(T)$ normalized to the number of photons incident on the surface of the sample. The next step is to discuss the influence of parameters from Eq. (1) on the form of the $I_{\mathrm{T}}(T)$ dependence.

\subsection{Triplet luminescence quenching}

In case both temperature-dependent factors of Eq. (1) decrease with an increasing of temperature, the $I_{\mathrm{T}}(T)$ function is characterized by a negative slope, wherein the quantum efficiency $\eta_{\mathrm{R}}(T)$ is decreasing (see Figure 5, curve 1 ) within the range from 1 to $1 /\left(1+\delta P_{\mathrm{R}}\right)$. It has a minimum which depends on the ratio $p_{05} / P_{4}$. If $\delta$ $P_{\mathrm{R}} \rightarrow 0$, then $\eta_{\mathrm{T}}$ will weakly depend on the temperature, keeping a value that is very close to 1 over the entire temperature range. However, the situation $\delta P_{\mathrm{R}}>1$ is most often realized, since the radiative triplet-singlet transitions are spin forbidden [30], and their rate $P_{4}$ is less than the frequency factor $p_{05}$ for the nonradiative relaxation.

The ratio between activation barriers $E_{2}$ and $E_{3}$ determines the form of $\eta_{\text {ISC }}(T)$ function, which decreases ranging from 1 to $1 /\left(1+\delta P_{\text {ISC }}\right)$ if $\Delta E_{\text {ISC }}<0$. In this case,

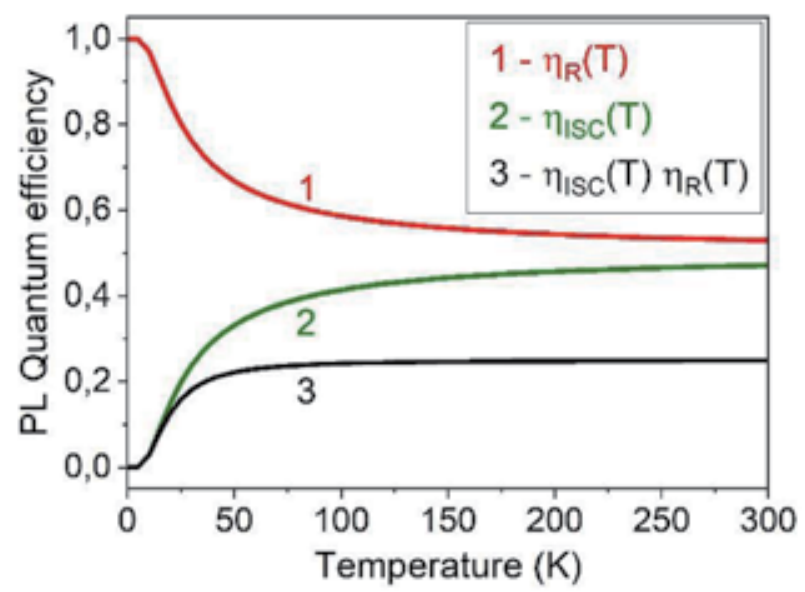

Figure 5 .

Calculated temperature dependences of the quantum efficiency factors for different processes: (1) triplet-singlet radiative transitions; (2) intersystem crossing; (3) two-step process $\eta_{I S C}(T) \cdot \eta_{R}(T)$. 
the form of $\eta_{\text {ISC }}(T)$ function is similar to that for $\eta_{R}(T)$, as was shown in Figure 5, curve 1 . Thus, the PL quenching curve will have an asymptote corresponding to a certain value $I_{\infty}$ (Figure 6, curve 1). If $\Delta E_{\mathrm{ISC}}=0$, the first exponent in Eq. (1) is equal to 1 , which causes a temperature independence of $\eta_{\text {ISC }}$ quantum efficiency. For this case, the $I_{\mathrm{T}}(T)$ dependence can be described by the Mott function [31] (Figure 6, curve 2), and quenching of triplet PL occurs due to the nonradiative transition 5 (Figure 4).

The $I_{\infty}$ value is determined by the parameters of the competing processes. The $I_{\infty}$ value increases if $\delta P_{\text {ISC }}$ and $\delta P_{\mathrm{R}}$ kinetic factors decrease. It should be noted that the $I_{\infty}$ parameter is some hypothetical constant, because the quenching at high temperatures isn't considered in the model of direct excitation. In fact, the $I_{\mathrm{T}}(T)$ dependences will tend to zero in the range of high temperatures. However, in some cases the experimental $I_{\mathrm{T}}(T)$ curves can have a saturation region at room temperature $[10,12,13]$. The PL intensity in the saturation region can be contingently accepted as $I_{\infty}$ value. This helps to simplify the mathematical processing.

\subsection{Triplet luminescence growth}

For the case of triplet PL growth, the $\eta_{\mathrm{ISC}}(T)$ function has an increasing character because the $\eta_{R}(T)$ function definitely decreases. As Eq. (1) shows, the quantum efficiency of the intersystem crossing $\left(\eta_{\text {ISC }}\right)$ increases with increasing of temperature if $\Delta E_{\text {ISC }}>0$ (see Figure 5, curve 2), wherein the intensity of triplet PL increases up to the $I_{\infty}$ value (Figure 6 , curve 4 ) or the $I_{\mathrm{T}}(T)$ curve has a maximum (see Figure 6, curve 5). If the maximum is absent, the occupation of the triplet state predominates over the process of the luminescence quenching. It is possible, when $\mathrm{d} I_{\mathrm{T}} / \mathrm{d} T>0$ condition is realized, which can be transformed as:

$$
\eta_{\mathrm{ISC}}(T) \delta P_{\mathrm{ISC}}\left(\Delta E_{\mathrm{ISC}}\right) \exp \left(\frac{\Delta E_{\mathrm{ISC}}}{k T}\right)>\eta_{\mathrm{R}}(T) \delta P_{\mathrm{R}} E_{\mathrm{Q}} \exp \left(-\frac{E_{\mathrm{Q}}}{k T}\right)
$$

After some mathematical transformations. we can write:

$$
n>l x+m,
$$

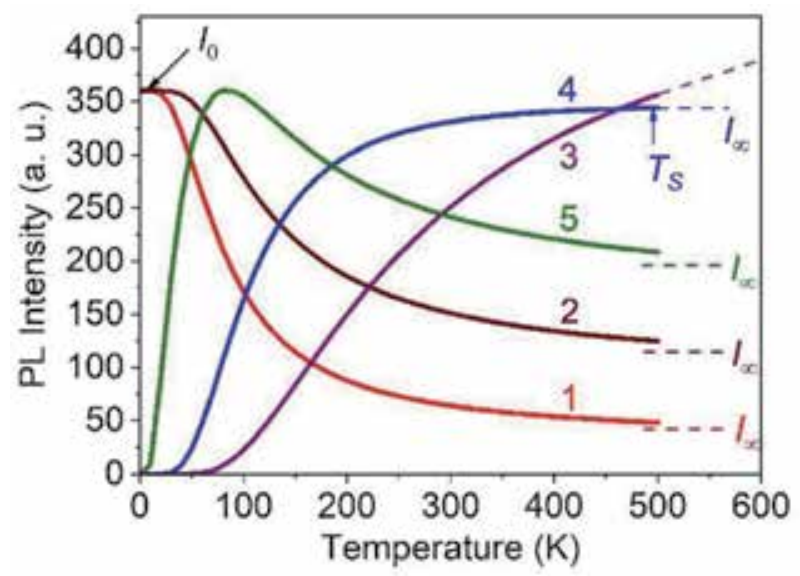

Figure 6.

PL temperature dependences for different ratios of energy factor $\Delta \mathrm{E}_{I S C}$ of intersystem crossing and PL quenching thermo-activation barrier $\mathrm{E}_{Q}:$ (1) $\Delta \mathrm{E}_{I S C}<0$; (2) $\Delta \mathrm{E}_{I S C}=0$; (3) $\Delta \mathrm{E}_{I S C}>C \cdot \mathrm{E}_{Q}$; (4) $\Delta \mathrm{E}_{I S C}=\mathrm{C} \cdot \mathrm{E}_{Q}$; (5) $0<\Delta \mathrm{E}_{I S C}<\mathrm{C} \cdot \mathrm{E}_{Q}$. 
where

$$
x=\exp \left(\frac{\Delta E_{\mathrm{ISC}}}{k T}\right) ; n=\frac{E_{\mathrm{Q}}}{\Delta E_{\mathrm{ISC}}}+1 ; l=\delta P_{\mathrm{R}} \cdot \frac{E_{\mathrm{Q}}-\Delta E_{\mathrm{ISC}}}{\Delta E_{\mathrm{ISC}}} ; m=\frac{\delta P_{\mathrm{R}}}{\delta P_{\mathrm{ISC}}} \cdot \frac{E_{\mathrm{Q}}}{\Delta E_{\mathrm{ISC}}}
$$

Since $\mathrm{n}>1$, finally one can obtain the following:

$$
\Delta E_{\mathrm{ISC}}>C \cdot E_{\mathrm{Q}}
$$

where

$$
C=\left(\frac{1}{\delta P_{\mathrm{ISC}}}+1\right)\left(\frac{1}{\delta P_{\mathrm{R}}}+1\right)^{-1}
$$

The inequality (Eq. (7)) shows that in general the temperature dependence $I_{\mathrm{T}}(T)$ is determined by both the peculiarities of the QD energy structure and their vibrational properties. From Eq. (8) one can see that the coefficient $C$ is responsible for the relations between the kinetic factors $\left(\delta P_{\mathrm{ISC}}, \delta P_{\mathrm{R}}\right)$ for the radiative transitions and intersystem crossing. In the case of slow kinetics of PL $\left(\delta P_{\text {ISC }}>>1\right.$ and $\delta$ $P_{\mathrm{R}}>>1$ ) the $\mathrm{C} \rightarrow 1$, one can neglect the vibrational properties of the QDs.

If instead of inequality (Eq. (7)) we will deal with the strict equality $\Delta E_{\mathrm{ISC}}=C \cdot E_{\mathrm{Q}}$, the $I_{\mathrm{T}}(T)$ dependence will be presented as the function with saturation point at temperature $T_{\mathrm{S}}>\Delta E_{\mathrm{ISC}} / k$ (see Figure 6 , curve 4 ).

\subsection{Extremal temperature dependence of triplet luminescence}

The maximum for $I_{\mathrm{T}}(T)$ curve occurs under the condition that the derivative of (1) is equal to zero. Thus, the energy factor of the singlet-triplet intersystem crossing $\Delta E_{\mathrm{ISC}}$ and the activation barrier of quenching $\left(E_{\mathrm{Q}}\right)$ have the following relationship:

$$
0<\Delta E_{\mathrm{ISC}}<C \cdot E_{\mathrm{Q}}
$$

Previously we noted that $C$ coefficient is introduced for accounting the influence of the rate of the vibrational processes on the form of PL temperature dependence for the QDs. However, the triplet luminescence is characterized by a relatively slow kinetics, when the $C$ parameter is close to 1 . Thus, we can state the following key points:

1. Rapidly decreasing $I_{\mathrm{T}}(\mathrm{T})$ curves mean that the thermal activation barrier is higher than the activation energy for the intersystem crossing $\left(\Delta E_{\mathrm{ISC}}<0\right)$.

2. In the case of temperature-independent occupation of the triplet states $\left(\Delta E_{\mathrm{ISC}}=0\right)$, the PL quenching can be described by the Mott type function (see Figure 6, curve 2). The nonradiative triplet-singlet channel is responsible for this process (see Figure 4, transition 5).

3. If the intersystem crossing barrier is high $\left(\Delta E_{\mathrm{ISC}}>C \cdot E_{\mathrm{Q}}\right)$, the intensity of the triplet luminescence increases. In this case the efficiency of intersystem crossing reaches its saturation at high temperatures.

4. The luminescence intensity increases until some point of saturation at temperature $T_{\mathrm{S}}$ when $\Delta E_{\mathrm{ISC}}=C \cdot E_{\mathrm{Q}}$. This saturation point corresponds to the balance between the quenching process and the occupation of $\mathrm{T}_{1}$ triplet states. 
5. If $0<\Delta E_{\mathrm{ISC}}<C \cdot E_{\mathrm{Q}}$, the curve $I_{\mathrm{T}}(T)$ has an extremum. In the range of $I_{\mathrm{T}}(T)$, increasing the occupation process of triplet states dominates. The decreasing of $\mathrm{PL}$ intensity is due to the prevalence of the luminescence quenching.

6. The shape of the $I_{\mathrm{T}}(T)$ dependence isn't affected by the vibrational properties of the luminescent QDs $(C \approx 1)$, if the PL kinetic is relatively slow (kinetic factors $\delta P_{\mathrm{ISC}}$ and $\delta P_{\mathrm{R}}$ significantly greater than 1$)$.

Analysis of Eqs. (2) and (3) can be performed in a similar way. These analytical equations describe the temperature behavior of the QD triplet luminescence at excitation within the spectral range of the exciton absorption in a wide bandgap matrix. As shown by Eq. (2), the simplified two-step model of this process corresponds to the decreasing function of the first type (see Figure 6, curve 1). All of the five above mentioned types of $I_{\mathrm{T}}(T)$ can be described by Eq. (3) for different $E_{\mathrm{Q}}, E_{\mathrm{ST}}$, and $\Delta E_{\mathrm{oc}}$ values. The main condition for increasing of this function is $\Delta E_{\mathrm{oc}}>0$.

Summarizing, the $\delta P_{\mathrm{T}}$ and $\Delta E_{\mathrm{ST}}$ parameters characterizing the occupation of radiative states are the key parameters for the approximation of the PL temperature dependence for QDs. The values of these parameters differ for the direct and indirect excitation mechanisms. Herewith, for all three models, transition 4 is the same, so one can fix the parameters $\delta P_{\mathrm{R}}$ and $E_{\mathrm{Q}}$ in the approximation operation.

\section{Theory and experiment comparison}

\subsection{A case of direct excitation}

In order to check the adequacy of the model for direct excitation (Eq. (1)), we have performed the analysis of the experimental PL temperature dependences for silicon QDs with the well-known luminescence properties [4, 5, 12, 13, 31]. There are selective PL bands at 1.7-1.8 eV with a full-width maximum at half-height (FWHM) of $0.18-0.15 \mathrm{eV}$ at direct excitation.

Figure 7 (curves 1 and 2) shows the experimental temperature dependences $I_{T}(\mathrm{~T})$, constructed from the integrated intensities of the Si QD luminescence bands excited by the radiation $3.6 \mathrm{eV}$ of nitrogen laser in the temperature range 9-300 K. To clarify the effect of the energy parameters $E_{\mathrm{ISC}}$ and $E_{\mathrm{Q}}$ on the shape and the luminescence intensity, we built simulated curves $I_{\mathrm{T}}(T)$, which are presented in Figure $7 \mathbf{b}$.

In Table 1 the calculated parameters of considered PL temperature dependences (curves 3-7) are listed. Table 1 and Figure 7 show that increase of $\Delta E_{\text {ISC }}$ causes the reducing of PL intensity due to the reducing of the efficiency of intersystem crossing. On the contrary, increase of the activation barrier $E_{\mathrm{Q}}$ for the competing process leads to an increase in the PL intensity due to the decrease of the quenching effectiveness. For all cases, the increasing of the activation energies leads to an increasing of the Tm temperature, which corresponds to the maximum of $I_{\mathrm{T}}(T)$ curve.

As was discussed in the section "Extremal temperature dependence for the triplet luminescence," all calculated curves except curve 5 belong to the fifth type of form. Curves 4 and 7 have the maxima outside the temperature range, which is shown in Figure 7. Curve 5 corresponds to the form of the third type. From Table 1, it is seen that this curve satisfies the condition (Eq. (7)).

The third-type curves demonstrate the lowest PL intensity. In the framework of developing the effective nanophosphors, the materials with the fifth-type form of 


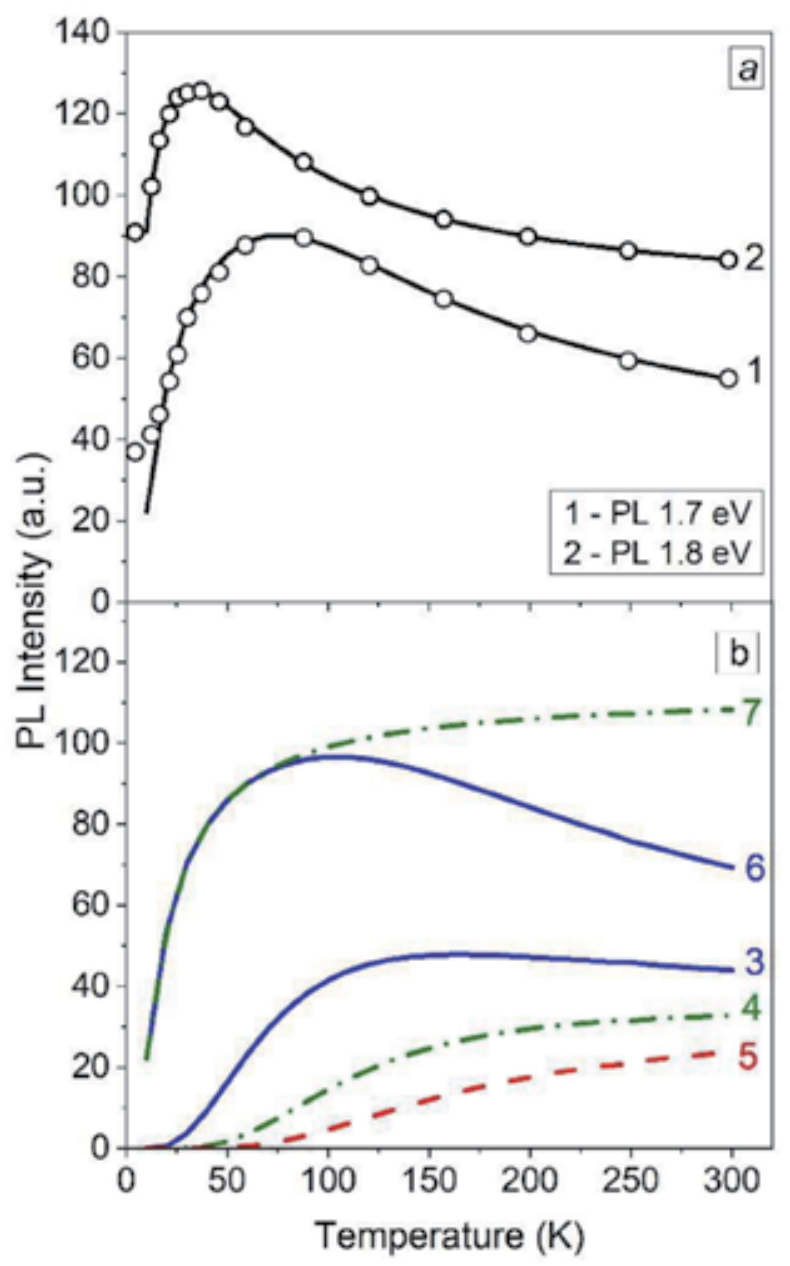

Figure 7.

$P L$ intensity at directly excited Si QDs versus temperature in silica films implanted by Si and $C$ ions (a): (1) $-\mathrm{SiO}_{2} / \mathrm{Si}$ (emission $\left.1.7 \mathrm{eV}\right) ;(2)-\mathrm{SiO}_{2} / \mathrm{Si} / \mathrm{C}($ emission $1.8 \mathrm{eV}) ;(b)-$ simulated curves $\mathrm{I}_{T}(\mathrm{~T})$, obtained by using the Eq. (1).

PL temperature dependence (curves 6 and 7 in Figure 7) are of a great interest. Thus, both high intensity and stability of PL for a wide temperature range can be achieved if the activation energy $E_{\mathrm{Q}}$ of the PL quenching increases. The main attention was paid to the relationship between the activation energies of the nonradiative processes on the basis of the analysis of Eq. (1) and the parameters, which impact on the form of experimental PL quenching curves. However, it must not be neglected that the kinetic parameters of the emission centers also influence the shape of the temperature behavior and intensity of PL.

The PL intensity additionally increases, if the pre-exponential factors $\delta P_{\text {ISC }}$ and $\delta P_{\mathrm{R}}$ decreases, as shown by Eq. (1). If the corresponding parameters will be tenfold different $\left(p_{02}>10 p_{03}\right.$ and $\left.P_{4}>10 p_{05}\right)$, this effect will be more noticeable.

However, such relationships are unlikely in the case of slow kinetics of the triplet luminescence. At the same time, the PL intensity can decrease due to the influence of reducing factors, such as the ratio of the kinetic parameters. So, a wider series of real experimental dependencies should be considered and analyzed. It allows to 


\begin{tabular}{ccccc}
\hline Curves & $\Delta E_{I S C}, \mathrm{eV}$ & $E_{Q}, \mathrm{eV}$ & $C \cdot E_{Q}, \mathrm{eV}$ & $T_{m}, \mathrm{~K}$ \\
\hline 1 & 0.0020 & 0.0260 & 0.0294 & 33 \\
\hline 2 & 0.0007 & 0.0090 & 0.0090 & 75 \\
\hline 3 & 0.0100 & 0.0260 & 0.0294 & 166 \\
\hline 4 & 0.0200 & & & 461 \\
\hline 5 & 0.0300 & & & - \\
\hline 6 & 0.0020 & 0.0400 & 0.0452 & 104 \\
\hline 7 & & 0.2000 & 0.2260 & 378 \\
\hline
\end{tabular}

Table 1.

Energy and kinetic parameters of the nonradiative processes for the curves $I_{T}(T)$ in Figure 7. Comment: For strings 1 and $3-7$, the correction is $C=1.13\left(\delta P_{I S C}=1.80, \delta P_{R}=2.65\right)$; for string $2 C=1\left(\delta P_{I S C}=1.10, \delta P_{R}=\right.$ 1.09); the intensity scale parameter is $I o=317$ a.u.

determine which model parameters are more prone to changes in the characteristics of QDs and how they respond to these changes.

To solve this task, we considered the experimental results on PL of Si QDs in $\mathrm{SiO}_{2}$ films obtained by Wang et al. [12]. In this work, authors obtained the Si QDs with different sizes by using the various doses of silicon ions. The emission bands are red-shifted from 1.65 to $1.43 \mathrm{eV}$ with an increasing of ion dose. In addition, the broadening of emission bands from 0.2 to $0.4 \mathrm{eV}$ was observed. However, the emission bands experienced a slight red shift $(<0.1 \mathrm{eV})$ and a change in width $(<0.05 \mathrm{eV})$ with an increasing of temperature.

The temperature dependences of the luminescence and their approximation by using Eq. (1) are shown in Figure 8. It is seen that the form of quenching curves depends on the ion fluence. On the one hand, epy curves 1 and 2 show a monotonic increase at low fluence. On the other hand, curves 3 and 4 have maxima around 158 and $163 \mathrm{~K}$, respectively, at high fluence. Herewith, all temperature dependences correspond to the fifth type, as was shown by results of fitting (Figure 8 and Table 2; see also "Extremal temperature dependence for the triplet luminescence" section). Maxima of curves 1 and 2 are observed outside the given temperature range. The larger $\Delta E_{\text {ISC }}$ energy factor of the intersystem crossing compared to this parameter for curves 3 and 4 explains their high-temperature position. The position of the temperature maximum is also affected by the activation barrier $E_{\mathrm{Q}}$ for the triplet-singlet PL quenching. The maximum is shifted to the high-temperature region if the barrier increases (Table 2 ). This effect is especially pronounced for the amorphous clusters.

In addition, a change in the ion fluence also impacts the kinetic factors of the exciton relaxation, which is reflected in the PL intensity. In particular, there is a significant reduction in the conversion kinetic factor $\delta P_{\text {ISC }}$ (Table 2, pp. 3 and 4) in the initial stages of exposure, which leads to a strong increase in the PL intensity (see Figure 8, curves 1 and 2). At higher ion fluences, there is increasing of the radiation kinetic factor $\delta P_{\mathrm{R}}$ (Table 2, pp. 5 and 6), which results in the decreasing of the PL intensity (see Figure 8, curves 3 and 4), wherein the relaxation processes, which compete with the triplet PL, are characterized by a faster kinetics $\left(\delta P_{\text {ISC }}>1\right.$ and $\delta P_{\mathrm{R}}>1$ ). From Table 2 one can see that the corresponding kinetic factors are varied in the values that range between 1.5 and 7 .

For all curves the correction parameter $C$ is close to 1 (Figures 7 and 8 ). It means the energy parameters have a great impact on the form of PL quenching curve. This conclusion is made on the basis of our theoretical $(C=1-1.13)$ evaluations and experimental results $(C=0.85-1.24)$ obtained in the work [12]. 


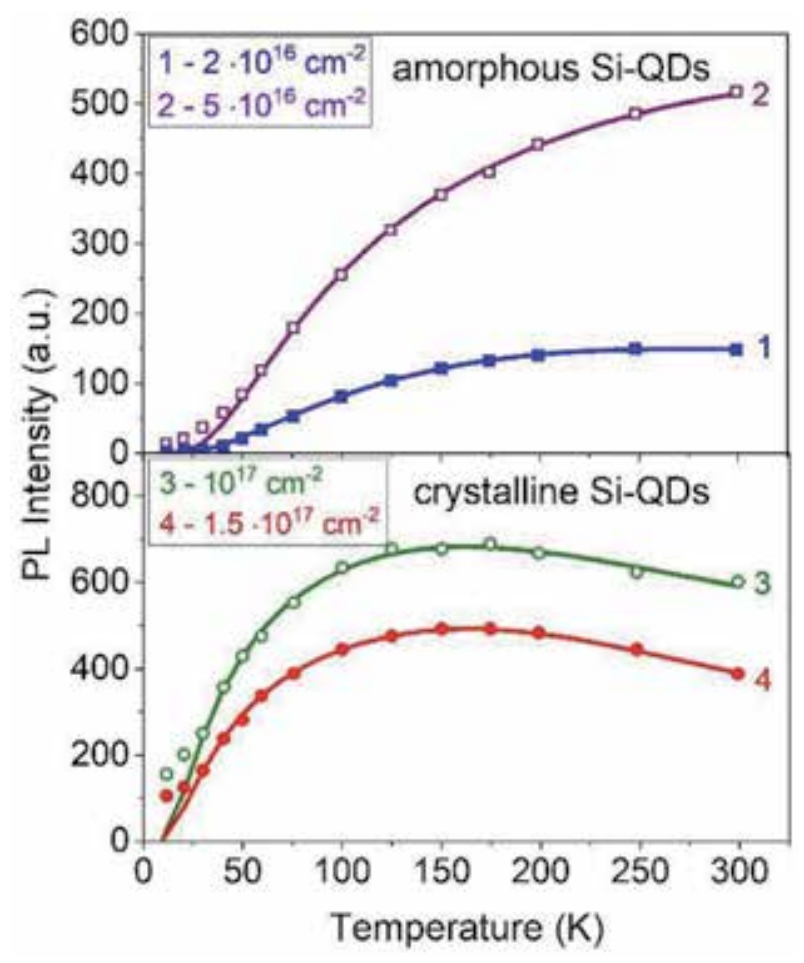

Figure 8.

$P L$ intensity at directly excited $\mathrm{Si} Q D$ s versus temperature in $\mathrm{SiO}_{2} /$ Si films implanted with different ion fluences. The circles denote an experiment [12], while the curves represent the calculated data using Eq. (1). Amorphous Si-clusters, curves $(1,2)$; crystalline Si-clusters, curves $(3,4)$. The excitation by argon laser $(2.4 \mathrm{eV})$.

\begin{tabular}{lcccccccc}
\hline QD-kind & $\mathbf{h} \nu_{\mathrm{PL}}, \mathrm{eV}$ & $\boldsymbol{\delta} \boldsymbol{P}_{I S C}$ & $\boldsymbol{\delta} \boldsymbol{P}_{\boldsymbol{R}}$ & $\mathrm{C}$ & $\Delta E_{I S C}, \mathrm{eV}$ & $\boldsymbol{E}_{\boldsymbol{Q}}, \mathrm{eV}$ & $\boldsymbol{C} \cdot \boldsymbol{E}_{\boldsymbol{Q}}, \mathrm{eV}$ & $\boldsymbol{T}_{\boldsymbol{m}}, \mathrm{K}$ \\
\hline \multirow{2}{*}{ Amorphous } & 1.8 & 1.10 & 1.09 & 1.00 & 0.0007 & 0.0090 & 0.0090 & 33 \\
\cline { 2 - 8 } & 1.7 & 1.80 & 2.65 & 1.13 & 0.0017 & 0.0258 & 0.0292 & 75 \\
\cline { 2 - 8 } & 1.65 & 7.10 & 2.91 & 0.85 & 0.0100 & 0.0640 & 0.0544 & 271 \\
\hline \multirow{2}{*}{ Crystalline } & 1.6 & 2.12 & 2.06 & 0.99 & 0.0110 & 0.1290 & 0.1277 & 438 \\
& 1.5 & 1.50 & 2.80 & 1.23 & 0.0040 & 0.0540 & 0.0664 & 158 \\
& 1.43 & 2.40 & 7.20 & 1.24 & 0.0050 & 0.0710 & 0.0880 & 163 \\
\hline
\end{tabular}

Table 2.

The approximation results of temperature dependencies for the directly excited PL in Si QDs at implanted $\mathrm{SiO}_{2}$ films.

\subsection{A case of indirect excitation}

The low density of the QD electronic states and the high density of matrix states cause a low probability for direct excitation of QDs PL by high-energy photons $(10-12 \mathrm{eV})$. By this reason, the analytical processing of PL temperature dependence for QDs should be performed with Eq. (2) or Eq. (3).

To check the models describing the indirect QD PL excitation, we have used the experimental data of QD luminescence under the synchrotron excitation $(11.6 \mathrm{eV})$. The luminescence of $\mathrm{Si}$ QDs in amorphous $\mathrm{SiO}_{2}$ films is observed at $1.8 \mathrm{eV}$ $(\mathrm{FWHM}=0.15 \mathrm{eV})[4,5]$. Figure 9 shows the temperature dependences of the integrated PL intensity (circles). 
The results of using Eqs. (2) and (3) for approximation are listed in Table 3. The OriginPro software was used for analytical processing of the obtained data and for determining the errors associated with the parameters of model. The analytical dependences of the three-stage $\left(I_{\mathrm{T}}{ }^{\prime \prime}(T)\right)$ and two-stage $\left(I_{\mathrm{T}}{ }^{\prime}(T)\right)$ processes are shown in Figure 9 as solid and dashed lines, respectively. The fitting error $(0.3-0.8 \%)$ does not exceed the measurement error ( $\sim 2 \%)$ for both cases. It means the two models are in good agreement with the experimental data for investigated temperature range. So, preferred models should be selected, taking into account experimental conditions and physical considerations regarding the energy structure of the $\mathrm{SiO}_{2}$ matrix and nanoparticles.

On the basis of experimental results, the PL quenching for Si QDs starts from the liquid-helium temperature, as shown in Figure 9. A characteristic plateau at $100-160 \mathrm{~K}$ temperatures is in a good agreement with the chosen models for the indirect excitation and confirms the non-elementary mechanism of the process. The form of experimental PL quenching curve indicates the barrier-free $\left(E_{7}=0\right)$ character for the transfer of the excitation energy from $\mathrm{SiO}_{2}$ matrix states to $\mathrm{Si}$ QDs in accordance with the two-stage scheme (Eq. (2)). The decreasing dependences $I_{\mathrm{T}}$ " $(T)$ show not only the barrier-free excitation transfer $\left(E_{10}=0\right)$ but also the negative energy factor of population of $\mathrm{T}_{1}$ states $\left(\Delta E_{\mathrm{oc}}<0\right)$ in accordance with the three-stage scheme (Eq. (3)).

Transitions 10 and 11 (Figure 4) in the total scheme of the population process of levels $\mathrm{T}_{1}$ can be ignored for the case of the thin-film $\mathrm{SiO}_{2}$ matrix implanted with silicon and carbon ions, as was proven by the acceptable accuracy of the two-stage model. On the contrary, the energy factor of population of states $\mathrm{T}_{1}\left(\Delta E_{\mathrm{oc}}\right)$ has a low absolute value, as was shown in Table 3. As noted above, this condition excludes the contribution of highly excited states to the kinetics of thermal relaxation of confined excitons. So, for the describing of PL temperature behavior for $\mathrm{Si}$ QDs in $\mathrm{SiO}_{2}$ host at indirect excitation, the two-stage model is quite an acceptable mathematical tool.

The value of the kinetic factor $\left(\delta P_{\mathrm{T}}\right)$ (Table 3$)$ shows that the energy transfer to the $\mathrm{QD}$ occurs relatively slowly. The frequency factor $\left(p_{08}\right)$ of the exciton selftrapping is $125-150$ times larger than the rate of this process. Due to the relatively

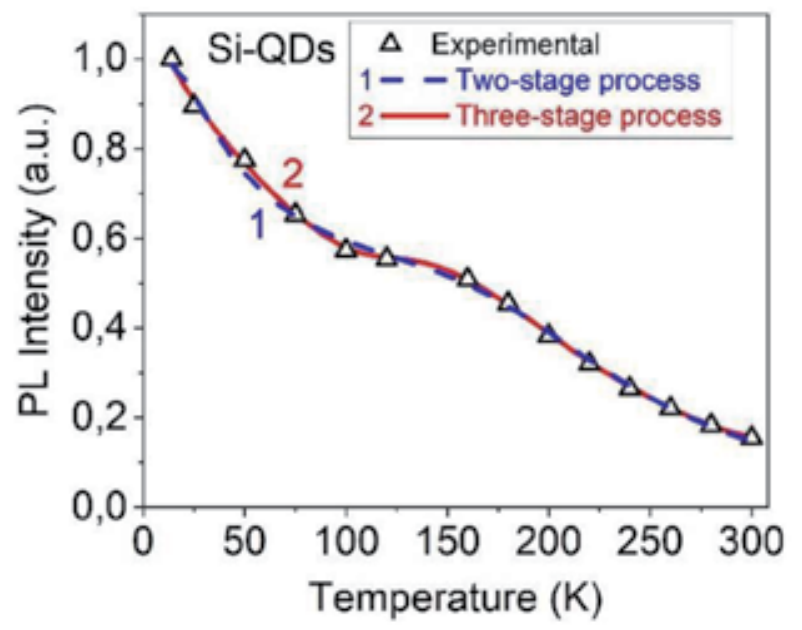

Figure 9.

$P L$ intensity at indirectly excited amorphous $\mathrm{Si}$ QDs versus temperature in $\mathrm{SiO}_{2} / \mathrm{Si} / \mathrm{C}$ films. The trianglesExperimental data (emission at $1.8 \mathrm{eV}$, excitation at $11.6 \mathrm{eV}$ ) $[4,5]$. A dashed line denotes Eq. (2) for the two-step process. The solid line represents $\mathrm{Eq}$. (3) for the three-step process. 


\begin{tabular}{lccc}
\hline Characteristics & & Two-stage model & Three-stage model \\
\hline Self-trapping barrier of FE & $E_{S T}, \mathrm{eV}$ & 0.104 & 0.110 \\
\hline Energy factor of $\mathrm{T}_{1}$ occupation & $\Delta E_{O C}, \mathrm{eV}$ & - & -0.002 \\
\hline Barrier of PL quenching & $E_{Q}, \mathrm{eV}$ & 0.006 & 0.009 \\
\hline Transfer factor of kinetic energy & $\delta P_{T}{ }^{*}$ & 125.51 & 153.07 \\
\hline Kinetic factor of T1 occupation & $\delta P_{O C}$ & - & 0.36 \\
\hline Kinetic factor for PL radiative & $\delta P_{R}$ & 1.36 & 1.09 \\
\hline${ }^{*}$ For the two-step process, $\delta \mathrm{P}_{T}$ corresponds to transition 7 (Figure 4) and for the three-step process-transition 10.
\end{tabular}

Table 3.

Results of approximation for the PL temperature dependences under direct and indirect excitations of Si QDs in $\mathrm{SiO}_{2}$ films.

high activation barrier $\left(E_{\mathrm{ST}}=101-110 \mathrm{meV}\right)$ for self-trapping of the free excitons in the matrix (Table 3), the effective transfer of excitation FE $\rightarrow$ QDs is possible only at low temperatures. The efficiency of generation of the self-trapped excitons increases with an increasing of the temperature. As a consequence, the intensity of QD PL strongly decreases due to the additional quenching, appreciable at $T>150 \mathrm{~K}$ (Figure 9).

The obtained results show that the multistage relaxation of excited states at indirect excitation causes the complex character of the PL temperature dependence for QDs. So, one can ignore the participation of the highly excited states of the confined exciton and consider the two relaxation stages for the analysis of the mechanism of indirect excitation of $\mathrm{Si}$ QDs in the $\mathrm{SiO}_{2}$ matrix [6].

In addition, the excitation method of QD luminescence also affects significantly the form of PL quenching curve. Depending on the type of excitation (direct or indirect), the form of the PL quenching curve changes dramatically. On the basis of comparison of the experimental curves (Figures 7 and 9), we can assume that indirect energy transfer occurs directly on the triplet state of QD by passing through the singlet state. In another case, the stage of intersystem crossing should be included in the transition scheme. Then there is also the increasing region in the PL temperature dependence at indirect excitation.

\section{Dependence of the PL kinetic and energy parameters on structural and dimensional factors}

In the present study, we examined the temperature dependences of the Si QD PL experimentally and observed that the energy radiative transitions are different (Figures 7 and 8). It's known that the spectral shift of emission band for QDs is inversely proportional to the square of its radius $\left(\Delta h \nu \sim R^{-2}\right)[1-3,32,33]$. On the basis of this relationship, we estimate the size of Si QDs, as listed in Table 2. The diameter of luminescent QDs is about 3.6-5.4 $\mathrm{nm}$. This assessment is consistent with the previous literature [12].

The high-resolution transmission electron microscopy (HRTEM) shows that when the samples are implanted by Si ions with the fluences between $1.5 \cdot 10^{17} \mathrm{~cm}^{-2}$ and $10^{17} \mathrm{~cm}^{-2}$, the average size of nanocrystal is between $3.8 \pm 1.2 \mathrm{~nm}$ and $3.5 \pm 1.5 \mathrm{~nm}$, respectively (Figure 10). Based on the fact that the radius of exciton in the bulk silicon [3] is about $4.2-4.9 \mathrm{~nm}$, we assume that all the samples are subjected to the effect of strong quantum confinement. 
(a)

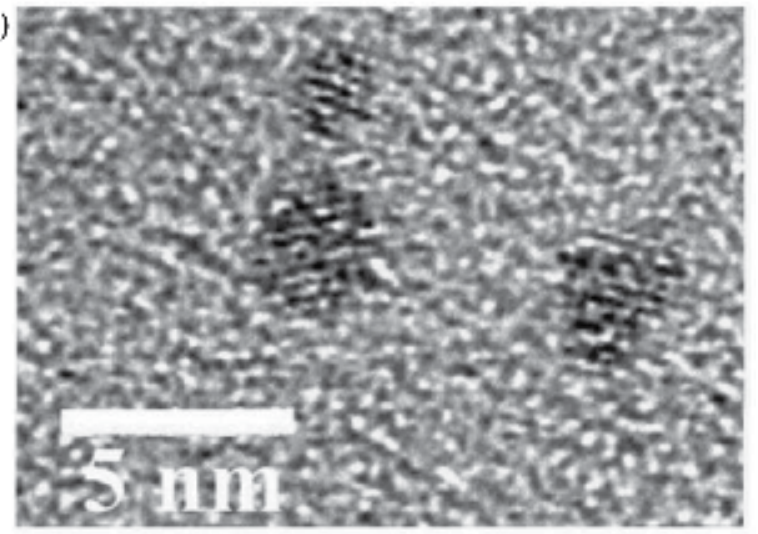

(b)

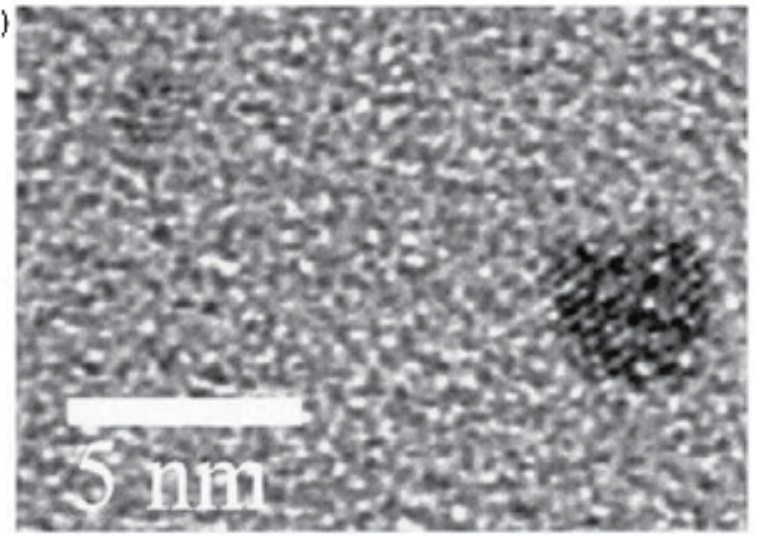

Figure 10.

HRTEM images of lattice fringe of $\mathrm{SiO}_{2}$ films (250 nm), implanted by Si-ions; (a) fluence $1.510^{17} \mathrm{~cm}^{-2}$, (b) $10^{17} \mathrm{~cm}^{-2}[12]$.

However, according to the diffraction data, the nanoparticles differ in size and internal structure. Figure 11(a) and (b) clearly confirms the presence of Si crystals in samples implanted by ions with fluences A and B; however, SAD does not show any sign of diffraction rings originating from anything but amorphous $\mathrm{SiO}_{2}$ in Figure 11(c) and (d). Studies have shown that the PL bands with maxima at 1.8 $-1.6 \mathrm{eV}$ are found for the amorphous Si nanoparticles, while the emission bands at $1.5-1.43 \mathrm{eV}$ are related with the crystalline Si nanoparticles $[4,5,10,12]$. Consequently, the change in the size, morphology, and structural ordering of QDs strongly affects the dependence of PL temperature curves on the ion fluence. In addition, the disorder degree in atomic structure and position of emission bands heavily depend on the size of QDs.

The form of the PL quenching curves is determined by the activation barriers and frequency factors, which are strongly affected by the all abovementioned factors (Figures 7 and 8 ).

The analysis of physical properties of the PL of Si QDs was performed on the basis of the data listed in Table 2. So, when the size of QDs increases:

1. The extremum of the PL quenching curve shifts to the range of higher temperatures.

2. The value of parameter $\Delta E_{\text {ISC }}$ corresponding to the intersystem crossing of excitons increases (Figure 12, curve 1). 
3. The activation barrier $E_{Q}$ for the nonradiative relaxation increases (Figure 12, curve 2).

4. The kinetic factors of the intersystem crossing $\left(\delta P_{I S C}\right)$ and radiation $\left(\delta P_{R}\right)$ take a maximum value at $1.65 \mathrm{eV}$ for the case of amorphous $\mathrm{Si}$ nanoparticles. The intersystem crossing $\left(\delta P_{I S C}\right)$ and radiation $\left(\delta P_{R}\right)$ increase for the case of crystalline Si nanoparticles.

When the structure of Si QDs is transformed from amorphous to crystalline, a dramatic reduction in the thermal activation characteristics occurs. From Figure 12 we can see that after crystallization the transition energy parameters $\left(\Delta E_{\text {ISC }}\right.$ and $\left.E_{\mathrm{Q}}\right)$ are significantly reduced. Experimentally, this effect can be observed as the shift of the maximum for the $I_{\mathrm{T}}(T)$ curve from 438 to $158 \mathrm{~K}$ (Figure 8).

The right interpretation of the abovementioned key points is impossible without knowledge of system of configuration curves for the emission center. The following reasons can result in the changes of the kinetic parameters and the activation barriers for nonradiative transitions:

1. The terms (configuration curves) of exciton are shifted either on the energy scale or on the coordinate configuration scale.

(a)

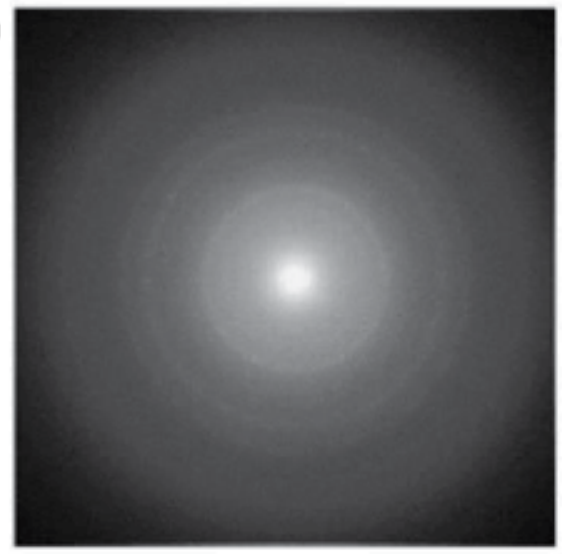

(c)

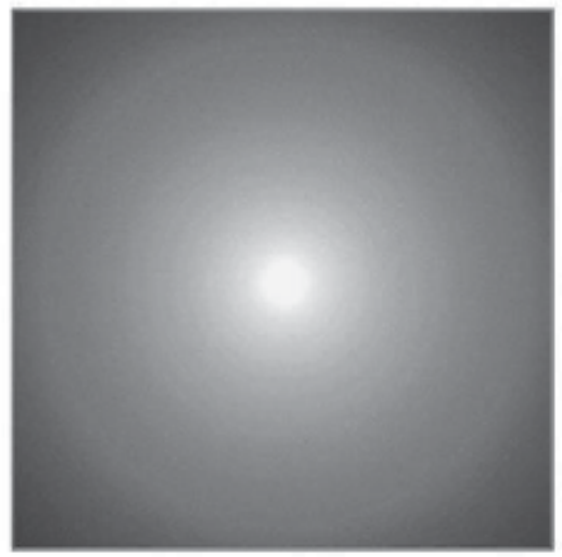

(b)

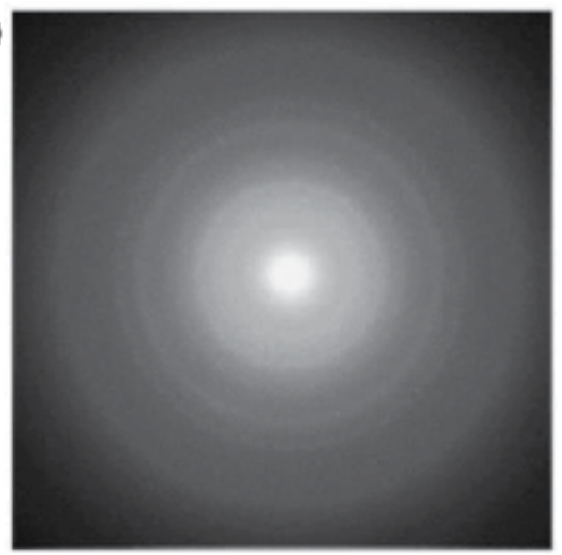

(d)

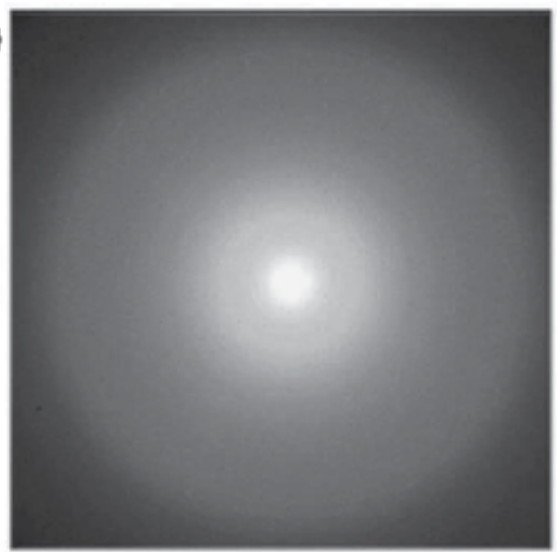

Figure 11.

Selected area diffraction (SAD) pattern images; (a) fluence $1.5 \times 10^{17} \mathrm{~cm}^{-2},(b) 10^{17} \mathrm{~cm}^{-2}$,

(c) $5 \times 10^{16} \mathrm{~cm}^{-2},(d) 2 \times 10^{16} \mathrm{~cm}^{-2}[12]$. 


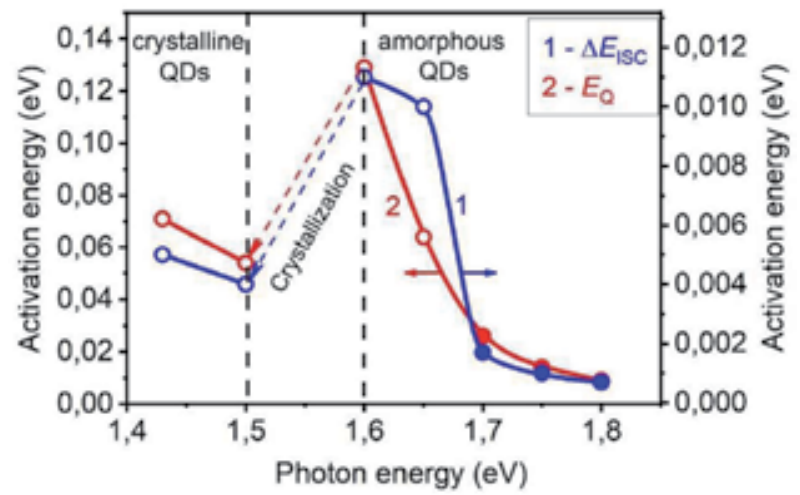

Figure 12.

The relationship between the energies of radiative PL transitions and the activation parameters of nonradiative transitions in silicon QDs in crystalline and amorphous state, which shows their dependence on structural and dimensional factors. The energy factor of the intercombining conversion is designated as $\mathrm{E}_{I S C}$, and the thermally activated barrier of PL quenching is $\mathrm{E}_{Q}$. the filled circles show the results of the data presented in $[4,5,10,13]$ and the open circles [12, 13].

2. The broadening or narrowing of configuration curves is observed.

3. The changes in the degree of continual disorder result in the distribution of configuration curves on the energy and configuration scales.

A system of hypothetical configuration curves with the terms of singlet ground state $\mathrm{S}_{0}$ and terms of singlet and triplet excited states $\left(\mathrm{S}_{1}\right.$ and $\mathrm{T}_{1}$, respectively) is shown in Figure 13. For clarity, the distribution of terms caused by the continual disorder isn't shown. It is assumed that the parameters $\Delta E_{\mathrm{ISC}}$ and $E_{\mathrm{Q}}$ take some effective values, which correspond to the maxima of distribution functions.

The main observation of the reduction in size of nanoparticles is the increase of the optical transitions energy, owing to the shift of the terms relative to each other [1-3]. We assume that $S_{0}, S_{1}$, and $T_{1}$ have a parabolic form; we can see that the shift of $S_{1}$ to the position of $S_{1}$ ' will lead to a decrease in the energy factor $\Delta E_{\text {ISC }}$ of the intersystem crossing (see Figure 13a).

This is well consistent with the curve 1 of Figure 12. However, a similar shift of the position for $\mathrm{T}_{1}$ toward $\mathrm{T}_{1}$ ' (Figure 13b) implies that increasing the energy of the radiative transition $(h \nu)$ will increase the activation barrier $\left(E_{\mathrm{Q}}{ }^{\prime}\right)$. At the same time, according to the experimental results (see Figure 12, curve 2), this barrier has to be decreased. Figure 12 shows that this contradiction can be solved if the displacement of $\mathrm{T}_{1}$ is accompanied by its extension $\left(\mathrm{T}_{1}\right.$ ”). This effect corresponds to the decrease of the frequency factor $p_{05}$ and kinetic factor $\delta P_{\mathrm{R}}$ where the approximation results confirm the increasing of the energy of radiative transition (Table 2).

The abovementioned four key points were found experimentally $[4,5,12,13]$. There is a red shift of emission bands from 1.8 to $1.4 \mathrm{eV}$ with an increasing of QD size. In addition, the PL band broadening is observed $(0.15-0.4 \mathrm{eV})$, which can be caused by the broadening of the configuration curve for the $\mathrm{T}_{1}$ excited triplet state (Figure 13b).

In the case of amorphous nanoparticles (Figure 12), the increase of the effective parameters $\Delta E_{\mathrm{ISC}}$ and $E_{\mathrm{Q}}$ in a larger QD size can be due to the variations in the corresponding distribution functions of energy levels. Since the size effect in the amorphous nanoparticles manifests itself more strongly in comparison with the crystalline nanoparticles, we can assume the quite importance of the continual disordering in the formation of QD optical properties. It should be noted that there 


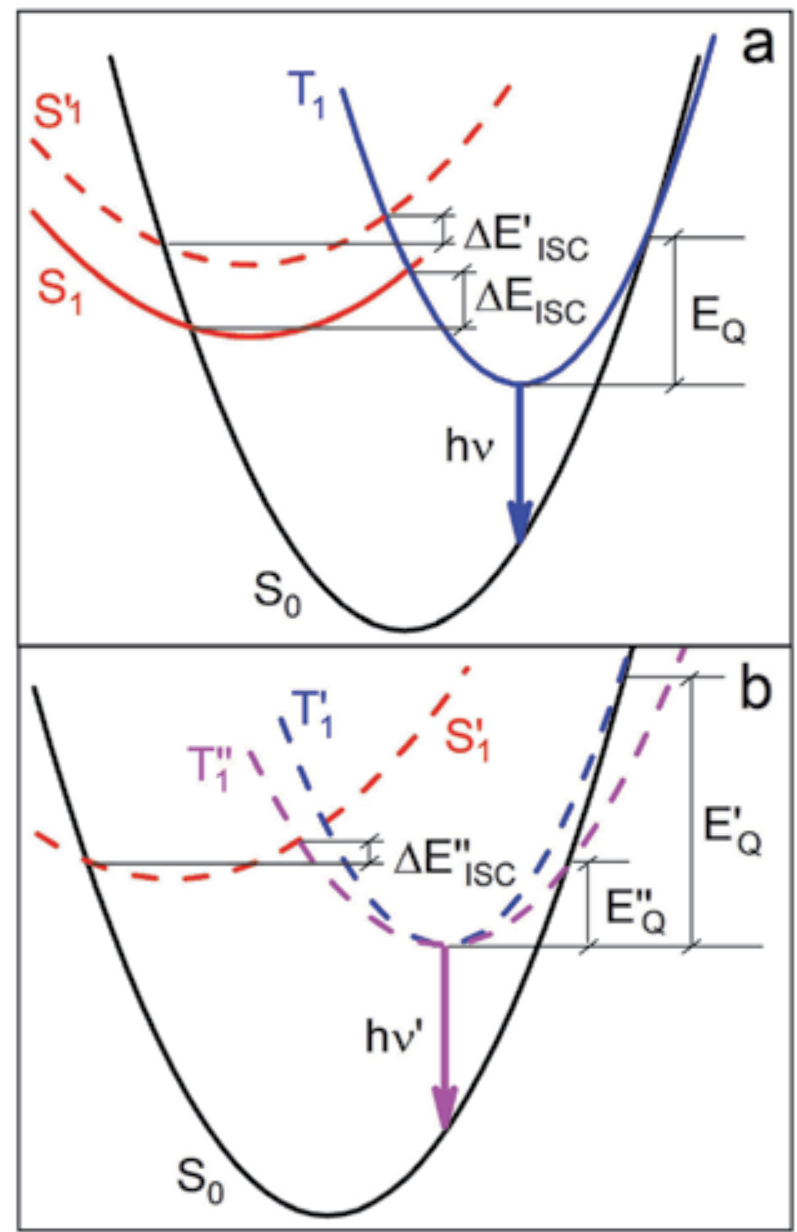

Figure 13.

Schematic illustration of the change in the energy factor $\Delta \mathrm{E}_{\mathrm{ISC}}$ of intercombination conversion (a) and the activation barrier $\mathrm{E}_{Q}$ of the PL quenching $(b)$ with a decrease in the size of quantum dots. The figure shows the initial terms of the ground and excited triplet and singlet states $\left(S_{o}, T_{1}, S_{1}\right)$, the displaced terms of the excited triplet and singlet states $\left(T_{1}, S_{1}{ }^{\prime}\right)$, and the shifted and broadened term of the excited triplet state $\left(T_{1}\right.$ ').

is a sharp decrease in activation barriers at transition from amorphous to crystalline QDs. From this point of view, the structural disorder is becoming a negligible factor. On the other hand, the dependence of shift and broadening of the configuration curves on the QD size also are observed for the case of crystalline QDs. Table 2 shows that the energy and the kinetic parameters of nonradiative transitions depend on the size of QDs.

The effect on structural and dimensional factors on the activation parameters of the radiative and nonradiative relaxations can be estimated from the three-level system of QD luminescence (Figure 13). However, for this task it is necessary to conduct special experiments and theoretical modeling (ab initio calculations). This issue is outside the scope of this work.

The developed ideas are a powerful tool for the control of the optical properties of QDs depending on the size, composition, and structure factors. We believe, the proposed approach is universal and can be applied to semiconductor QDs (such as $\mathrm{Si}, \mathrm{Ge}, \mathrm{C}$ ) in dielectric hosts. The estimation of the applicability of this model to QDs of another type (e.g., with complex composition or special structural features [34]) can be performed using a special experimental check. 


\section{Conclusion}

This chapter considers the temperature behavior of luminescent semiconductor quantum dots that have formed by thermal and ion beam methods. Analytical expressions characterizing the PL intensity of confined excitons were obtained on the basis of a generalized energy scheme for direct or indirect excitation of QDs.

Five types of temperature dependences of the luminescence of quantum dots were considered, and model parameters that affect the shape of the temperature curves were analyzed. The main variables of these models are the ratios of the rates of competing relaxation processes as well as the differences of their activation barriers. The derived expressions allow us to analyze and explain the experimentally observed luminescence temperature dependences of the most diverse form.

The models presented in this chapter were tested on the example of temperature dependences of PL silicon nanoclusters in silica matrix under different optical excitations. High informativeness and sensitivity of analytical expressions to dimensional effects and structural disordering in QDs substance are shown.

It was found that indirect excitation of $\mathrm{Si}$ QDs leads to a decreasing PL temperature dependence, which is due to a three-stage relaxation process. At the same time, the temperature dependences of PL upon direct excitation of silicon quantum dots are in the form of curves with a maximum and are characterized by a two-stage relaxation process. The increased effect of Si QD photoluminescence with increasing temperature, which is observed experimentally, can be explained by an increase in the density of triplet excitations acting as radiative states.

The observed structural and dimensional effects are explained using a hypothetical configurational scheme. We have found that the confined exciton effect in silicon nanoclusters manifests itself in the form of a decrease in the thermal activation barriers of nonradiative processes. Crystallization of the silicon amorphous nanoclusters also leads to a sharp decrease in the energy parameters of all thermally activated processes.

The data presented in this chapter can be used in the field of new functional materials and devices for nanophotonics. The developed ideas here can be considered as a tool for predicting and controlling the luminescent properties of quantum dots under their composition, size, and structural ordering change.

\section{Author details}

Anatoly Zatsepin and Dmitry Biryukov*

Institute of Physics and Technology, Ural Federal University, Yekaterinburg, Russia

*Address all correspondence to: a.f.zatsepin@urfu.ru; bir-70@list.ru

\section{IntechOpen}

(C) 2020 The Author(s). Licensee IntechOpen. This chapter is distributed under the terms of the Creative Commons Attribution License (http://creativecommons.org/licenses/ by/3.0), which permits unrestricted use, distribution, and reproduction in any medium, provided the original work is properly cited. (cc) BY 


\section{References}

[1] Zhang JZ. Optical Properties and Spectroscopy of Nanomaterials. New York: World Scientific Publishing Co Pte Ltd; 2009. p. 383

[2] Pei Y, Zeng XC. Investigating the structural evolution of thiolate protected gold clusters from firstprinciples. Nanoscale. 2012;4: 4054-4072. DOI: $10.1039 / \mathrm{c} 2 \mathrm{nr} 30685 \mathrm{a}$

[3] Oda S, Ferry D, editors. Silicon Nanoelectronics. Taylor \& Francis Group, LLC: Boca Raton; 2006. p. 310. DOI: $10.1201 / 9781420028645$

[4] Zatsepin AF, Buntov EA, Kortov VS, Tetelbaum DI, Mikhaylov AN, Belov AI. Mechanism of quantum dot luminescence excitation within implanted $\mathrm{SiO}_{2}: \mathrm{Si}: \mathrm{C}$ films. Journal of Physics: Condensed Matter. 2012;24:045301. DOI: 10.1088/ 0953-8984/24/4/045301

[5] Zatsepin AF, Buntov EA. Chapter 5. Synchrotron-excited photoluminescence spectroscopy of silicon- and carboncontaining quantum dots in low dimensional $\mathrm{SiO}_{2}$ matrices. In: SiliconBased Nanomaterials. New York, Heidelberg, Dordrecht, London: Springer, Springer Series in Materials Science; 2013. pp. 89-117. DOI: $10.1007 /$ 978-1-4614-8169-0

[6] Pan Z, Ueda A, Xu H, Hark SK, Morgan SH, Mu R. Photoluminescence of Er-doped $\mathrm{ZnO}$ nanoparticle films via direct and indirect excitation. Journal of Nanophotonics. 2012;6:063508. DOI: 10.1117/1.JNP.6.063508

[7] Reznitsky AN, Klochikhin AA, Permogorov SA. Temperature dependence of photoluminescence intensity of self-assembled CdTe quantum dots in the ZnTe matrix under different excitation conditions. Physics of the Solid State. 2012;54:123-133. DOI: $10.1134 /$ S1063783412010283
[8] Canham LT. Silicon quantum wire array fabrication by electrochemical dissolution of wafers. Applied Physics Letters. 1990;57:1046-1048. DOI: 10.1063/1.103561

[9] Nordin MN, Clowes J, Li SK, Curry RJ. Temperature dependent optical properties of $\mathrm{PbS}$ nanocrystals. Nanotechnology. 2012;23:275701. DOI: $10.1088 / 0957-4484 / 23 / 27 / 275701$

[10] Biryukov DY, Zatsepin AF. Analytical temperature dependence of the photoluminescence of semiconductor quantum dots. Physics of the Solid State. 2014;56:635-638. DOI: $10.1134 / \mathrm{S} 1063783414030056$

[11] Nagornykh SN, Pavlenkov VI, Mikhailov AN, Belov AI, Krasil'nikova LV, Kryzhkov DI, et al. Model of photoluminescence from ionsynthesized silicon nanocrystal arrays embedded in a silicon dioxide matrix. Technical Physics. The Russian Journal of Applied Physics. 2012;57:1672-1675. DOI: 10.1134/S1063784212120213

[12] Wang J, Righini M, Gnoli A, Foss S, Finstad T, Serincan U, et al. Thermal activation energy of crystal and amorphous nano-silicon in $\mathrm{SiO}_{2}$ matrix. Solid State Communications. 2008;147: 461-464. DOI: 10.1016/j.ssc.2008.07.011

[13] Zatsepin AF, Biryukov DY. The temperature behavior and mechanism of exciton luminescence in quantum dots. Physical Chemistry Chemical Physics. 2017;19:18721-18730. DOI: 10.1039/c7cp03357e

[14] Kapoor M, Singh VA, Johri GK. Origin of the anomalous temperature dependence of luminescence in semiconductor nanocrystallites. Physical Review B. 2000;61:1941-1945. DOI: 10.1103/PhysRevB.61.1941 
[15] Glinka YD, Lin SH, Hwang LP, Chen YT, Tolk NH. Size effect in selftrapped exciton photoluminescence from $\mathrm{SiO}_{2}$-based nanoscale materials. Physical Review B. 2001;64:085421. DOI: 10.1103/PhysRevB.64.085421

[16] Glinka YD, Lin SH, Chen YT. Time-resolved photoluminescence study of silica nanoparticles as compared to bulk type-III fused silica. Physical Review B. 2002;66: 035404. DOI: 10.1103/PhysRevB. 66.035404

[17] Kang Z, Liu Y, Tsang CHA, Ma DDD, Fan X, Wong N-B, et al. Water-soluble silicon quantum dots with wavelength-tunable photoluminescence. Advanced Materials. 2009;21(6):661-664. DOI: 10.1002/adma.200801642

[18] Erogbogbo F, Chang C-W, May JL, Liu L, Kumar R, Law W-C, et al. Bioconjugation of luminescent silicon quantum dots to gadolinium ions for bioimaging applications. Nanoscale. 2012;4:5483-5489. DOI: 10.1039/ c2nr31002c

[19] Guruvenket S, Hoey JM, Anderson KJ, Frohlich MT, Krishnan R, Sivaguru J, et al. Synthesis of silicon quantum dots using cyclohexasilane $\mathrm{Si}_{6} \mathrm{H}_{12}$. Journal of Materials Chemistry C. 2016;4:8206-8213. DOI: $10.1039 / \mathrm{c} 6 \mathrm{tc} 01435 \mathrm{f}$

[20] Roy D, Majhi K, Mondal MK, Saha SK, Sinha S, Chowdhury P. Silicon quantum dot-based fluorescent probe: Synthesis characterization and recognition of thiocyanate in human blood. ACS Omega. 2018;3(7):

7613-7620. DOI: $10.1021 /$ acsomega. $8 \mathrm{~b} 00844$

[21] Askari S, Macias-Montero M, Velusamy T, Maguire P, Svrcek V, Mariotti D. Silicon-based quantum dots: Synthesis, surface and composition tuning with atmospheric pressure plasmas. Journal of Physics D: Applied
Physics. 2015;48:314002. DOI: 10.1088/ 0022-3727/48/31/314002

[22] Gan J, Li Q, Hu Z, Yu W, Gao K, Sun J, et al. Study on phase separation in $\mathrm{a}^{-\mathrm{SiO}_{\mathrm{X}}}$ for $\mathrm{Si}$ nanocrystal formation through the correlation of photoluminescence with structural and optical properties. Applied Surface Science. 2011;257(14):6145-6151. DOI: 10.1016/j.apsusc.2011.02.019

[23] Zatsepin AF, Buntov EA, Zatsepin DA, Kurmaev EZ, Pustovarov VA, Ershov AV, et al. Energy band gaps and excited states in $\mathrm{Si} \mathrm{QD} / \mathrm{SiO}_{\mathrm{X}} / \mathrm{R}_{\mathrm{Y}} \mathrm{O}_{\mathrm{Z}}(\mathrm{R}=\mathrm{Si}, \mathrm{Al}, \mathrm{Zr})$ suboxide superlattices. Journal of Physics: Condensed Matter. 2019; 31(41):415301. DOI: $10.1088 /$ 1361-648X/ab30d6

[24] Ershov AV, Chugrov IA, Tetelbaum DI, Mashin AI, Pavlov DA, Nezhdanov AV, et al. Thermal evolution of the morphology, structure, and optical properties of multilayer nanoperiodic systems produced by the vacuum evaporation of $\mathrm{SiO}$ and $\mathrm{SiO}_{2}$. Semiconductors. 2013;47:481-486. DOI: 10.1134/S1063782613040064

[25] Zatsepin AF, Kuznetsova Yu A, Wong $\mathrm{CH}$. Creation of Si quantum dots in a silica matrix due to conversion of radiation defects under pulsed ion-beam exposure. Physical Chemistry Chemical Physics. 2019;21:25467-25473. DOI: 10.1039/c9cp04715h

[26] Zatsepin AF, Zatsepin DA, Boukhvalov DW, Gavrilov NV, Shur VY, Esin AA. The MROaccompanied modes of Re-implantation into $\mathrm{SiO}_{2}$-host matrix: XPS and DFT based scenarios. Journal of Alloys and Compounds. 2017;728:759-766. DOI: 10.1016/j.jallcom.2017.09.036

[27] Green RJ, Zatsepin DA, Onge DJS, Kurmaev EZ, Gavrilov NV, Zatsepin AF, et al. Electronic band gap reduction and intense luminescence in $\mathrm{Co}$ and $\mathrm{Mn}$ ion-implanted $\mathrm{SiO}_{2}$. Journal 
of Applied Physics. 2014;115:103708. DOI: $10.1063 / 1.4868297$

[28] Zatsepin AF, Pustovarov VA, Kortov VS, Buntov EA, Fitting HJ. Time-resolved photoluminescence of implanted $\mathrm{SiO}_{2}: \mathrm{Si}^{+}$films. Journal of Non-Crystalline Solids. 2009;355: 1119-1122. DOI: 10.1016/j. jnoncrysol.2009.01.048

[29] Zatsepin AF. Statics and dynamics of excited states of oxygen-deficient centers in $\mathrm{SiO}_{2}$. Physics of the Solid State. 2010;52:1176-1187. DOI: 10.1134/ S1063783410060107

[30] Sulimov VB, Sokolov VO, Dianov EM, Poumellec B. Photoinduced structural transformations in silica glass: The role of oxygen vacancies in the mechanism of formation of refractiveindex gratings by UV irradiation of optical fibres. Quantum Electronics. 1996;26:988-993. DOI: $10.1070 /$ QE1996v026n11ABEH000857

[31] Mott NF, Davis EA. Electronic Processes in Non-crystalline Materials. Oxford: Oxford University Press; 1979. p. 608

[32] Vaccaro L, Spallino L, Zatsepin AF, Buntov EA, Ershov AV, Grachev DA, et al. Photoluminescence of $\mathrm{Si}$ nanocrystals embedded in $\mathrm{SiO}_{2}$ : Excitation/emission mapping. Physica Status Solidi B: Basic Solid State Physics. 2015;252:600-606. DOI: 10.1002/ pssb.201451285

[33] De Boer WDAM, Timmerman D, Dohnalová K, Yassievich IN, Zhang H, Buma WJ, et al. Red spectral shift and enhanced quantum efficiency in phonon-free photoluminescence from silicon nanocrystals. Nature Nanotechnology. 2010;5:878-884. DOI: 10.1038/nnano.2010.236

[34] Pan SS, Li FD, Liu QW, Xu SC, Luo YY, Li GH. Strong localization induced anomalous temperature dependence exciton emission above 300
$\mathrm{K}$ from $\mathrm{SnO}_{2}$ quantum dots. Journal of Applied Physics. 2015;117:173101. DOI: 10.1063/1.4919595 